\title{
Journal of

\section{Mechanical failure of zigzag graphene nanoribbons under tensile strain induced by edge reconstruction}

\author{
Y. C. Cheng, Z. Y. Zhu and U. Schwingenschlögl* \\ Received 22nd June 2012, Accepted 28th September 2012 \\ DOI: 10.1039/c2jm34068b
}

\begin{abstract}
The structural and mechanical properties of graphene nanoribbons (GNRs) under uniaxial tensile strain are studied by density functional theory. The ideal strength of a zigzag GNR $(120 \mathrm{GPa})$ is close to that of pristine graphene. However, for a GNR with both edges reconstructed to pentagon-heptagon pairs (from hexagon-hexagon pairs) it decreases to $94 \mathrm{GPa}$ and the maximum tensile strain is reduced to $15 \%$. Our results constitute a comprehensive picture of the edge structure effect on the mechanical properties of GNRs.
\end{abstract}

\section{Introduction}

Graphene has attracted a lot of interest since it first became accessible experimentally in $2004 .{ }^{1}$ Recent experimental results ${ }^{2}$ have established graphene as the strongest material with the highest Young's modulus ( $\sim 1 \mathrm{TPa})$ ever measured. Its breaking strength of $\sim 130 \mathrm{GPa}$ represents the calculated intrinsic strength of a defect-free sheet. ${ }^{3}$ There are various theoretical studies about the mechanical properties of monolayer graphene suggesting that topological defects, such as monovacancies, Stone-Wales dislocations, slits, and holes, will weaken the strength. ${ }^{4,5}$ The Young's modulus of graphene decreases with the vacancy concentration and large slits or holes drastically reduce the fracture strength to $30-40 \mathrm{GPa}^{6}$

A substantial portion of the graphene studies is devoted to understanding the physics of graphene nanoribbons (GNRs), due to their potential use in electronic applications. As the typical width of a GNR is in the range of 4 to $20 \mathrm{~nm}$, the confinement effect opens a band gap, which is essential for room temperature transistor operation. ${ }^{7}$ It has been predicted that zigzag GNRs are semiconductors with two localized electronic edge states. These states are ferromagnetically ordered and couple to each other antiferromagnetically. When an external electric field is applied across the ribbon, half-metallicity can be realized in zigzag GNRs. $^{8}$

Various methods for obtaining GNRs have been demonstrated, such as lithographic patterning of graphene, ${ }^{9}$ chemical sonication, ${ }^{10}$ unzipping of multiwall carbon nanotubes, ${ }^{11}$ and bottom-up fabrication. ${ }^{12}$ Recently, Jin et $a l .{ }^{13}$ have obtained GNRs by fabricating two nearby holes in monolayer graphene, using prolonged electron irradiation. This tailors the width of GNRs from $2 \mathrm{~nm}$ down to even single $\mathrm{C}$ chains. When decreasing

Physical Sciences and Engineering Division, King Abdullah University of Science and Technology, Thuwal 23955-6900, Kingdom of Saudi Arabia. E-mail: udo.schwingenschlogl@kaust.edu.sa the GNR width, the edge plays an increasingly important role in the structural and mechanical properties. It has been proposed that the zigzag edge is metastable and a planar transition from hexagon-hexagon pairs to pentagon-heptagon pairs (StoneWales defect) spontaneously takes place at room temperature ${ }^{14}$ or high temperature. ${ }^{15}$ On the other hand, it has been demonstrated that the transition energy barrier is $1.12 \mathrm{eV}$ that is large enough to prevent transition from hexagon-hexagon to pentagon-heptagon pairs. ${ }^{16}$ However, the transition can occur under tensile strain larger than about $5 \%$ at room temperature. ${ }^{16}$ Reports on the mechanical properties of GNRs ${ }^{17-21}$ show that armchair and zigzag shaped edges decrease and increase the tensile strength, respectively. Therefore, it is important to achieve a comprehensive picture of the mechanical properties of GNRs and the effect of the edge reconstruction.

In this work, we investigate the mechanical properties of GNRs under uniaxial tensile strain, using calculations based on density functional theory. The influence of edge reconstruction on the mechanical properties is discussed in detail. The ideal strength of the zigzag GNR is close to that of pristine graphene. When the hexagon-hexagon pairs on the zigzag edge transform to pentagon-heptagon pairs, the ideal strength of the reconstructed GNR decreases to $94 \mathrm{GPa}$ and the maximum tensile strain is reduced to $15 \%$. The energetically favorable state of the zigzag GNR is anti-ferromagnetic for a tensile strain less than $5 \%$. Our study predicts the mechanism of the mechanical failure of the zigzag GNR under tensile strain, thus highlighting the importance of the edge structure for graphene based nanomechanical devices.

\section{Computational method}

Our calculations employ the generalized-gradient approximation of the exchange correlation functional with ultrasoft pseudopotentials, ${ }^{22}$ as implemented in the Quantum-ESPRESSO package. ${ }^{23}$ A high cut-off energy of $500 \mathrm{eV}$ and a mesh of $1 \times 8 \times$ $1 k$-points are used to achieve a high accuracy. Structural 


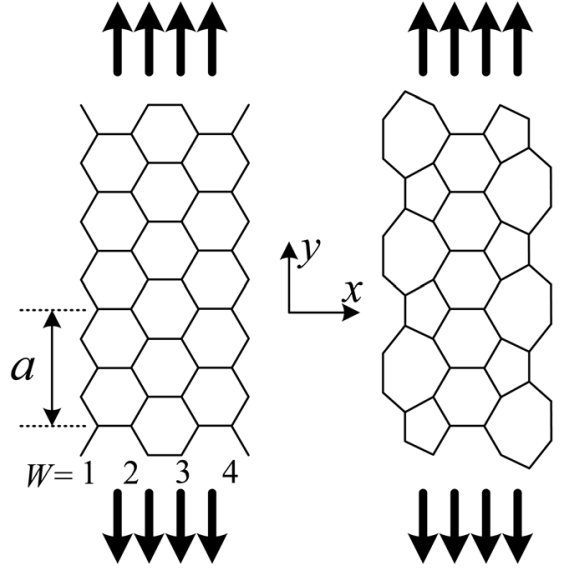

Fig. 1 Schematic structures of zigzag (left) and FR (right) GNRs with $w$ $=4$. The arrows denote the axis and the tensile strain direction.

optimization is carried out for all systems until the residual forces have converged to $0.003 \mathrm{eV} \AA^{-1}$. We describe the uniaxial strain along the edge direction ( $y$-axis) of the GNR (see Fig. 1) by $\varepsilon=$ $\left(a-a_{0}\right) / a_{0} \times 100 \%$, where $a$ and $a_{0}=4.92 \AA$ are the lattice parameters for the strained and pristine structure, respectively.

\section{Results and discussion}

Before addressing the mechanical properties of GNRs, we briefly discuss the electronic properties of zigzag GNRs under strain. The electronic properties of unstrained GNRs were studied extensively in the late $1990 s .{ }^{24,25}$ Fig. 2 presents the band structures and densities of states of zigzag GNRs in anti-ferromagnetic (AFM), ferromagnetic (FM), and non-magnetic (NM) states under a uniaxial tensile strain of $5 \%$. For the zigzag GNRs we find flat bands around the Fermi level due to the edge states. These flat bands result in sharp peaks in the density of states. In general, such van Hove singularities ${ }^{26}$ can result in density waves, ${ }^{27}$ magnetism, ${ }^{28}$ or even superconductivity. ${ }^{29}$ For the NM state, see Fig. 2(c), a sharp peak is located exactly at the Fermi level, yielding an electronic instability of the system. Spin splitting then results in magnetism. ${ }^{30}$ We find that the NM state of the zigzag GNR is unstable against both AFM and FM ordering. In the AFM state a band gap opens, whereas in the

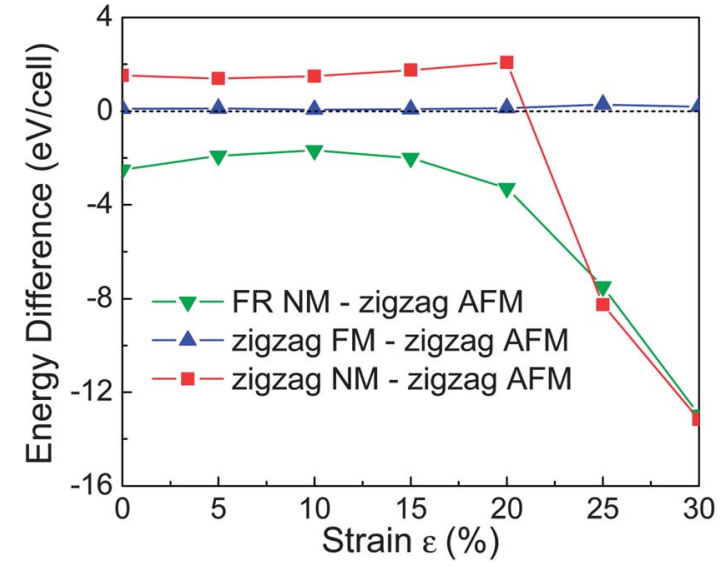

Fig. 3 Energy differences with respect to the AFM state of the zigzag GNR for the FR GNR in the NM state, the zigzag GNR in the FM state, and the zigzag GNR in the NM. The width is $w=4$ and uniaxial strain is applied along the edge direction ranging from $0 \%$ to $30 \%$.

FM state the Fermi level is located at the crossing point of two bands with almost linear dispersion and is far way from any flat band.

By calculating the energy difference between the zigzag GNRs in the AFM, FM, and NM states as well as the GNR with both edges reconstructed (FR), see Fig. 3, we demonstrate that the ground state of the zigzag GNR is AFM. We also conclude that the FR GNR is energetically favorable over the zigzag GNR under uniaxial strain ranging from $0 \%$ to $20 \%$. For the case of the zigzag GNR, the energy difference between the FM and AFM states is less than $0.1 \mathrm{eV}$ per cell, while the energy difference between the NM and AFM states is $\sim 1.5 \mathrm{eV}$ per cell. Moreover, the energy difference between the FM and AFM states is much smaller than the energy difference between the NM FR GNR and the AFM zigzag GNR ( $\sim 2.0 \mathrm{eV}$ per cell). Under uniaxial strain larger than $25 \%$, the NM configuration of the zigzag GNR becomes energetically favorable. There are few experimental observations of the FR GNR ${ }^{31}$ although it is theoretically demonstrated that this configuration is energetically favorable over the zigzag GNR. However, it is typical for carbon materials that there are many allotropes with large energy barriers that prevent transitions.
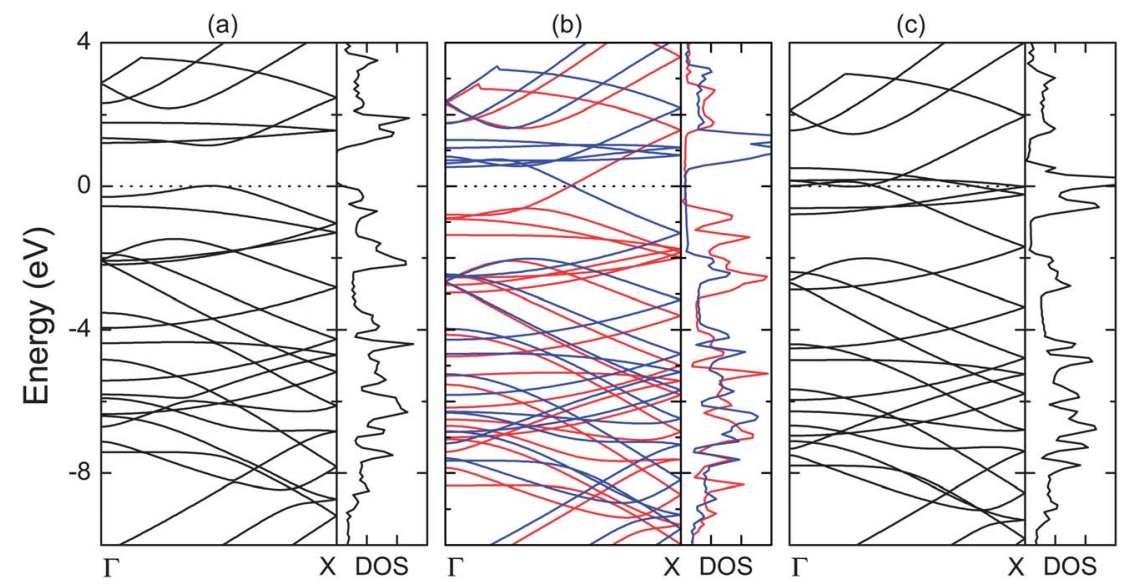

Fig. 2 Band structures and densities of states of zigzag GNRs in the (a) AFM, (b) FM, and (c) NM states. 


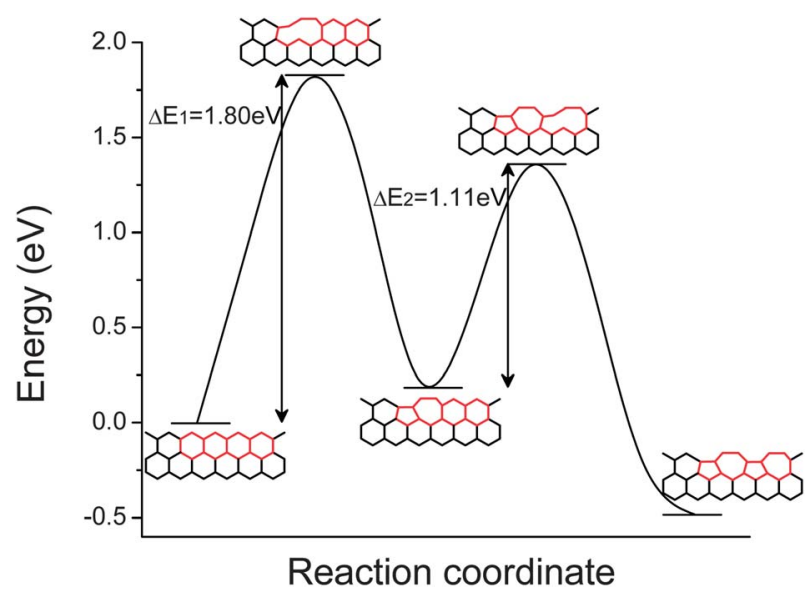

Fig. 4 Minimum energy path of the first and second step transitions from a hexagon-hexagon pair to a pentagon-heptagon pair.

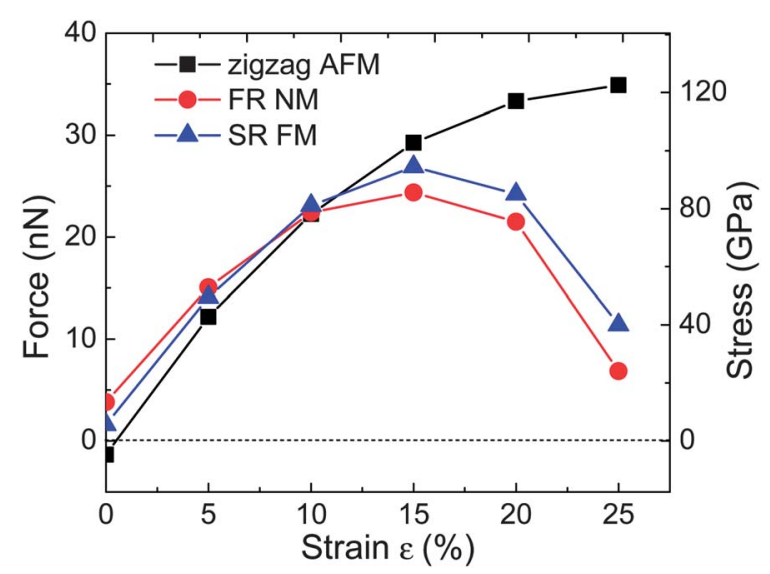

Fig. 5 Force-stress-strain relationships for zigzag, FR, and SR GNRs with a width of $w=4$ under uniaxial tensile strain along the edge. Force $F$ and stress $\sigma$ are connected by $\sigma=F / A$, with the area $A=19.38 \AA^{2}$.

In a previous work it has been found that the energy barrier for transition from a zigzag to a FR edge is $0.6 \mathrm{eV}$ and it has been claimed that the reconstruction takes place spontaneously at room temperature based on transition state theory. ${ }^{14}$ However, it has been pointed out that the first step of the reconstruction, i.e., the transformation of a pair of hexagons into a pentagonheptagon pair, is subject to a higher free energy barrier of $0.83 \mathrm{eV}$ at room temperature. ${ }^{15}$ This value was obtained by classical molecular dynamics calculations for a $w=6$ GNR with two free edges. In our present study we employ the nudged elastic band method $^{32}$ based on density functional theory to evaluate energy barriers. Our structure model is a $w=4$ GNR with one fixed edge. Our calculations result in energy barriers for the first and second transition steps of 1.80 and $1.11 \mathrm{eV}$, see Fig. 4. The discrepancy to ref. 15 can be attributed to the classical method employed there. Importantly, the fact that the energy barrier in our case is about $1 \mathrm{eV}$ larger prevents transition at room temperature. However, it is predicted that a zigzag GNR will transform into an FR GNR under uniaxial tensile strain larger than $5 \%$ at room temperature, because the transition barrier is lowered by the strain. ${ }^{16}$ Therefore, it is interesting to obtain insight into the effect of the edge reconstruction on the mechanical properties of a zigzag GNR.

Fig. 5 shows the ideal tensile strengths of the zigzag GNR, the GNR with only one edge reconstructed (SR), and the FR GNR with $w=4$. The ideal tensile strength is the highest achievable strength of a defect-free crystal under tensile strain at $0 \mathrm{~K} .{ }^{3}$ When the stress on the crystal is larger than the ideal tensile strength, the deformation of the crystal is irreversible. The latter is called mechanical failure. Ambiguity in the thicknesses and widths of ultra-thin GNRs suggests addressing the stress in terms of force $F$ (left $y$-axis; in $\mathrm{nN}$ ) rather than in terms of force per unit area, i.e., stress $\sigma$ (right $y$-axis; in $\mathrm{N} \mathrm{m}^{-2}$ or $\mathrm{Pa}$ ). ${ }^{33}$ Force and stress are connected to each other by the relationship $\sigma=F / A$ via the area $A$. To compare with previously reported ideal tensile strengths, we transform the one-dimensional force into a three-dimensional stress assuming that the effective thickness of graphene is $3.4 \AA$ and that the width is $5.7 \AA(w=4)$. This leads to $A=19.38 \AA^{2}$. The residual stresses of zigzag, $\mathrm{SR}$, and FR GNRs $(w=4)$ without tensile strain $\left(a=a_{0}\right)$ are $-4.8,13.3$, and 5.6 GPa, respectively. The residual stresses are edge stresses due to a lack of periodicity perpendicular to the edge direction. ${ }^{14-16}$ A series of incremental tensile strains have been applied along the edges. The ideal tensile strength of the zigzag GNR is about $120 \mathrm{GPa}$ at a strain of $\varepsilon=$ $25 \%$, which is consistent with previous reports. ${ }^{3,21}$ Moreover, the ideal tensile strengths of the SR and FR GNRs are about 94 and $85 \mathrm{GPa}$ at a strain of $\varepsilon=15 \%$, respectively. This indicates that the edge reconstruction weakens the ideal tensile strength, which leads to mechanical failure of the GNR at a strain of $\varepsilon>15 \%$. The consequence is a structural transition of the FR GNR, as reflected by imaginary frequencies in the phonon dispersion.

Fig. 6 and 7 show the phonon dispersion relationships for the zigzag GNR in the AFM and FM states $(w=4)$ under uniaxial

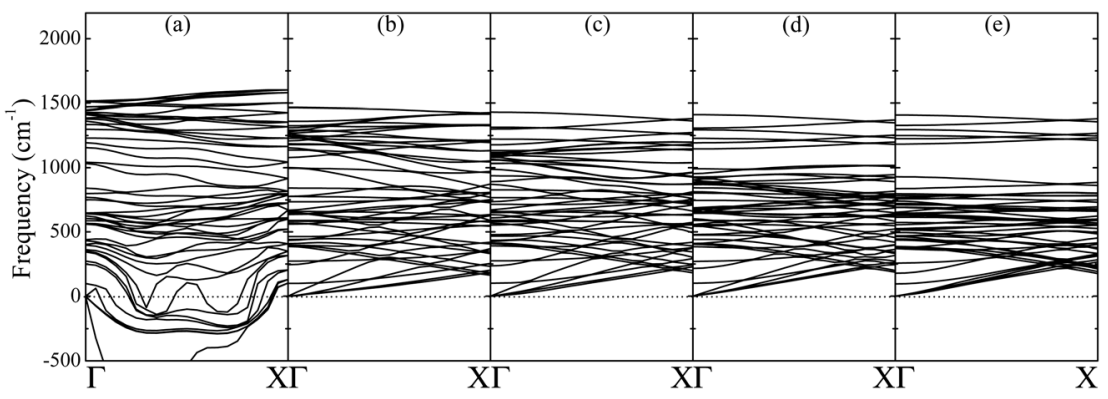

Fig. 6 Phonon dispersions for the zigzag GNR in the AFM state ( $w=4$ ) under a uniaxial tensile strain of (a) $0 \%$, (b) $5 \%$, (c) $10 \%$, (d) $15 \%$, and (e) $20 \%$. 


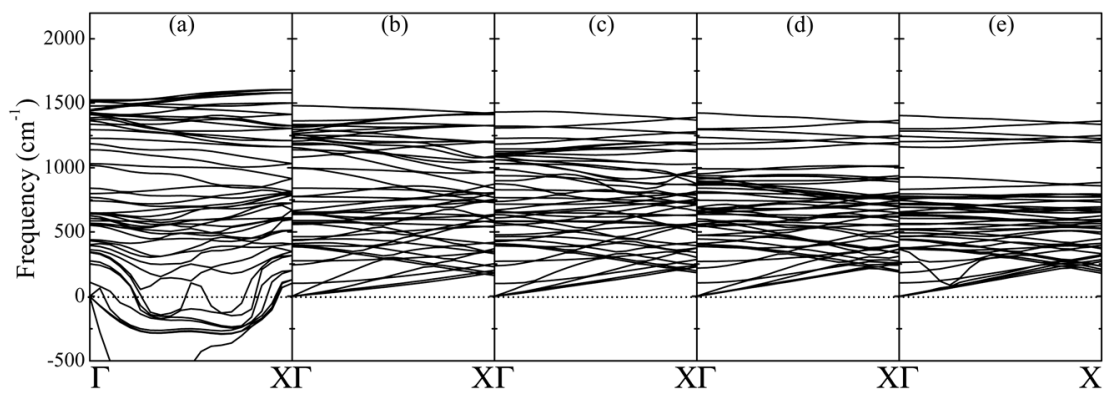

Fig. 7 Phonon dispersions for the zigzag GNR in the FM state $(w=4)$ under a uniaxial tensile strain of (a) $0 \%$, (b) $5 \%$, (c) $10 \%$, (d) $15 \%$, and (e) $20 \%$.

tensile strain ranging from $0 \%$ to $20 \%$, respectively. In general, the phonon dispersions of the two states are similar. Imaginary frequencies in Fig. 6(a) and 7(a) indicate instability of the structure without uniaxial tensile strain. The imaginary frequencies appear between the $\Gamma$ and $X$ points. Because of the residual edge stress for the zigzag GNR, a ripple is found without tensile strain. ${ }^{15,16}$ There is no imaginary frequency in the phonon dispersions for the zigzag GNR in the AFM and FM states under uniaxial strain ranging from $5 \%$ to $20 \%$. As discussed above, the AFM state is energetically more stable than the FM state under uniaxial tensile strain ranging from $0 \%$ to $20 \%$. The structural stability of the FM state is similar to that of the AFM state. Moreover, the phonon dispersion for the zigzag GNR in both the AFM and FM states under a uniaxial tensile strain of $15 \%$ and $20 \%$ shows an energy gap around $1000 \mathrm{~cm}^{-1}$.

Fig. 8 shows the phonon dispersion relationships for the FR GNR under uniaxial strain ranging from $0 \%$ to $20 \%$. The additional modes with frequencies around $2000 \mathrm{~cm}^{-1}$ correspond to in-plane vibrations along the edge of the two outermost $\mathrm{C}$ atoms of the heptagon. ${ }^{16}$ There is no imaginary frequency in the phonon dispersion relationships for the $w=4$ FR GNR, indicating stability under uniaxial tensile strain from $0 \%$ to $10 \%$, see Fig. $8(\mathrm{a}-\mathrm{c})$. Fig. $8(\mathrm{~d}$ and e) show an instability for uniaxial strain larger than $15 \%$, suggesting a structural transition of the FR GNR. The energy difference between the zigzag and FR GNRs in Fig. 3 shows that the zigzag GNR in the AFM state is less stable than the FR GNR under a uniaxial strain larger than $20 \%$. However, there is an abrupt energy decrease for the zigzag GNR in the NM state when the strain exceeds $20 \%$. In addition, the energy for the zigzag GNR in the NM state is slightly smaller than that for the FR GNR in the NM state. By checking the relaxed structure of the zigzag and FR GNRs in the NM state, we find that the heptagons, hexagons, or pentagons break, leading to carbon chain formation, which is consistent with previous results obtained by molecular dynamics. ${ }^{19,34}$

The edge of the zigzag GNR is reactive due to the dangling bonds. In the present study we assume that the GNR is in vacuum to prevent a reaction. However, in air the edge will be chemically saturated by water vapor, oxygen, etc. In previous studies on the structural and mechanical properties of the zigzag GNR, the dangling bonds on the zigzag edge were saturated by $\mathrm{H}$, O, or other chemical groups. ${ }^{14,17,19-21}$ The mechanical properties were found to be similar to those without saturation. ${ }^{21}$ However, saturation will prevent transition from the hexagon-hexagon pair to the pentagon-heptagon pair. We therefore can expect that it will conserve the inherent strength of the GNR. Also, for a realistic GNR there will be many kinds of defects on the edge, which induce extra stress and consequently affect the strength. The unit cell chosen for modeling the zigzag GNR in ref. 17-21 contains one hexagon along the edge direction, which is half of the unit cell of our calculation. Omitting the edge transition therefore has induced mechanical failure in these studies.

It has been discussed earlier that the first step of the transition from a hexagon-hexagon to a pentagon-heptagon pair has the higher transition barrier, see Fig. 4, while the second step is easier to achieve due to the smaller transition barrier. As a consequence, the hexagon-hexagon pairs of the zigzag edge transform progressively during the transition process towards an FR GNR. This result is consistent with previous findings from molecular dynamics simulations. ${ }^{15}$ This transition leads to a mixture of heptagons, hexagons, and pentagons. Consequently, the ideal strength of the GNR will depend on the concentration of the pentagon-heptagon pairs. In addition, once a hexagon-hexagon pair transforms under certain tensile strain, the externally applied tensile stress is partially canceled by the residual stress induced by the transformation. This is attributed to be the main reason for the mechanical failure of the FR GNR. We have only

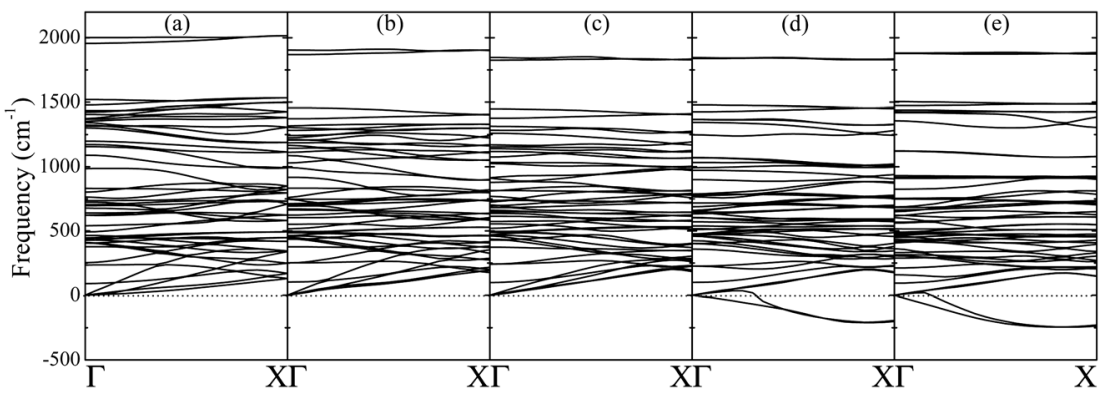

Fig. 8 Phonon dispersions for the FR GNR in the NM state $(w=4)$ under a uniaxial tensile strain of (a) $0 \%$, (b) $5 \%$, (c) $10 \%$, (d) $15 \%$, and (e) $20 \%$. 
discussed the effect of the hexagon-hexagon to the pentagonheptagon transformation on the mechanical properties of the zigzag GNR. The theoretically predicted hexagon-hexagonhexagon to heptagon-pentagon-heptagon group transformation on the edge of the armchair GNR illustrates that the transformation can also induce residual stress. ${ }^{35}$ Therefore, it is expected that mechanical failure affects the transformed armchair GNR. According to the transition state theory, increasing the temperature will increase the probability of edge transition. ${ }^{14-16}$ It is expected that at low temperatures the zigzag GNR can maintain a high mechanical strength. In contrast, at high temperatures a transition will occur and mechanical failure will be observed. Therefore, in experiments the stress-strain relationship of zigzag GNRs depends on the temperature.

According to Fig. 4, the saddle point of the transition from a hexagon-hexagon pair to a pentagon-heptagon pair coincides with the $\mathrm{C}-\mathrm{C}$ bond breaking perpendicular to the edge direction. After the breakage, the outermost $\mathrm{C}$ atom moves to one side and forces the neighboring $\mathrm{C}$ atom in the hexagon to move to the same side, leading to the formation of a pentagon-heptagon pair. The first transition will lead to a local tensile strain on neighboring hexagon-hexagon pairs. The phonon dispersions in Fig. 6-8 demonstrate mode softening when GNRs are subject to tensile strain, indicating that the strength of the $\mathrm{C}-\mathrm{C}$ bonds is lowered. Thus, the local tensile strain induced by the first transition weakens the $\mathrm{C}-\mathrm{C}$ bonds of neighboring hexagons. As a consequence, the energy barrier of the second transition is lower than that of the first.

\section{Conclusion}

In conclusion, we have investigated the structural and mechanical properties of zigzag and FR GNRs under uniaxial tensile strain by density functional theory. The ideal strength of the zigzag GNR is about $120 \mathrm{GPa}$, which is close to that of pristine graphene. In addition, the zigzag GNR is stable under uniaxial tensile strain up to $20 \%$ for both the AFM and FM states. When the hexagon-hexagon pair on the zigzag edge transforms to a pentagon-heptagon pair, the ideal strength of the reconstructed GNR decreases to $94 \mathrm{GPa}$, and the maximum tensile strain is reduced to $15 \%$.

\section{References}

1 K. S. Novoselov, A. K. Geim, S. V. Morozov, D. Jiang, Y. Zhang, S. V. Dubonos, I. V. Grigorieva and A. A. Firsov, Science, 2004, 306, 666.

2 C. Lee, X. Wei, J. W. Kysar and J. Hone, Science, 2008, 321, 385.

3 F. Liu, P. Ming and J. Li, Phys. Rev. B: Condens. Matter Mater. Phys., 2007, 76, 064120.

4 R. Khare, S. Mielke, J. Paci, S. Zhang, R. Ballarini, G. Schatz and T. Belytschko, Phys. Rev. B: Condens. Matter Mater. Phys., 2007, 75, 075412 .
5 O. V. Yazyev and S. G. Louie, Phys. Rev. B: Condens. Matter Mater. Phys., 2010, 81, 195420.

6 F. Hao, D. Fang and Z. Xu, Appl. Phys. Lett., 2011, 99, 041901.

7 X. R. Wang, Y. J. Ouyang, X. L. Li, H. L. Wang, J. Guo and H. J. Dai, Phys. Rev. Lett., 2008, 100, 206803.

8 E.-J. Kan, Z. Y. Li, J. L. Yang and J. G. Hou, Appl. Phys. Lett., 2007, 91, 243116.

9 M. Han, B. Özyilmaz, Y. Zhang and P. Kim, Phys. Rev. Lett., 2007, 98, 206805.

10 X. Li, X. Wang, L. Zhang, S. Lee and H. Dai, Science, 2008, 319, 1229.

11 L. Jiao, L. Zhang, X. Wang, G. Diankov and H. Dai, Nature, 2009, 458, 877.

12 J. Cai, P. Ruffieux, R. Jaafar, M. Bieri, T. Braun, S. Blankenburg, M. Muoth, A. P. Seitsonen, M. Saleh, X. Feng, K. Mullen and R. Fasel, Nature, 2010, 466, 470.

13 C. Jin, H. Lan, L. Peng, K. Suenaga and S. Iijima, Phys. Rev. Lett., 2009, 102, 205501.

14 P. Koskinen, S. Malola and H. Häkkinen, Phys. Rev. Lett., 2008, 101, 115502.

15 J. H. Kroes, M. Akhukov, J. Los, N. Pineau and A. Fasolino, Phys. Rev. B: Condens. Matter Mater. Phys., 2011, 83, 165411.

16 Y. C. Cheng, H. T. Wang, Z. Y. Zhu, Y. H. Zhu, Y. Han, X. X. Zhang and U. Schwingenschlögl, Phys. Rev. B: Condens. Matter Mater. Phys., 2012, 85, 073406.

17 R. Faccio, P. A. Denis, H. Pardo, C. Goyenola and A. W. Mombrú, J. Phys.: Condens. Matter, 2009, 21, 285304.

18 H. Zhao, K. Min and N. R. Aluru, Nano Lett., 2009, 9, 3012.

19 H. Bu, Y. Chen, M. Zou, H. Yi, K. Bi and Z. Ni, Phys. Lett. A, 2009, 373,3359 .

20 C. D. Zeinalipour-Yazdiand and C. Christofides, J. Appl. Phys., 2009, 106, 054318.

21 S.-S. Yu, C. Wang, W.-T. Zheng and Q. Jiang, Phys. Status Solidi A, 2011, 208, 2328.

22 D. Vanderbilt, Phys. Rev. B: Condens. Matter Mater. Phys., 1990, 41, 7892.

23 P. Giannozzi, S. Baroni, N. Bonini, M. Calandra, R. Car, C. Cavazzoni, D. Ceresoli, G. L. Chiarotti, M. Cococcioni, I. Dabo, A. D. Corso, S. deGironcoli, S. Fabris, G. Fratesi, R. Gebauer, U. Gerstmann, C. Gougoussis, A. Kokalj, M. Lazzeri, L. Martin-Samos, N. Marzari, F. Mauri, R. Mazzarello, S. Paolini, A. Pasquarello, L. Paulatto, C. Sbraccia, S. Scandolo, G. Sclauzero, A. P. Seitsonen, A. Smogunov, P. Umari and R. M. Wentzcovitch, J. Phys.: Condens. Matter, 2009, 21, 395502.

24 M. Fujita, K. Wakabayashi, K. Nakada and K. Kusakabe, J. Phys. Soc. Jpn., 1996, 65, 1920.

25 K. Wakabayashi, M. Fujita, H. Ajiki and M. Sigrist, Phys. Rev. B: Condens. Matter Mater. Phys., 1999, 59, 8271.

26 L. van Hove, Phys. Rev. B: Condens. Matter Mater. Phys., 1953, 89, 1189.

27 T. M. Rice and G. K. Scott, Phys. Rev. Lett., 1975, 35, 120.

28 M. Fleck, A. M. Oleś and L. Hedin, Phys. Rev. B: Condens. Matter Mater. Phys., 1997, 56, 3159.

29 W. Kohn and J. M. Luttinger, Phys. Rev. Lett., 1965, 15, 524.

30 D.-E. Jiang, B. G. Sumpter and S. Dai, J. Chem. Phys., 2007, 126, 134701.

31 P. Koskinen, S. Malola and H. Häkkinen, Phys. Rev. B: Condens. Matter Mater. Phys., 2009, 80, 073401.

32 G. Henkelman and H. Jonsson, J. Chem. Phys., 2000, 113, 9978.

33 A. K. Nair, S. W. Cranford and M. J. Buehler, Europhys. Lett., 2011, 95, 16002 .

34 J. Zhang, J. Zhao and J. Lu, ACS Nano, 2012, 6, 2704.

35 B. Huang, M. Liu, N. Su, J. Wu, W. Duan, B.-L. Gu and F. Liu, Phys. Rev. Lett., 2009, 102, 166404. 